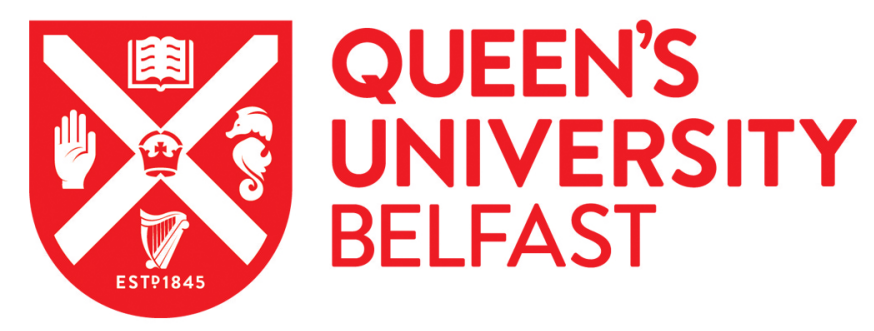

\title{
Propagation of nonclassical correlations across a quantum spin chain
}

Campbell, S., Apollaro, T. J. G., Di Franco, C., Banchi, L., Cuccoli, A., Vaia, R., Plastina, F., \& Paternostro, M. (2011). Propagation of nonclassical correlations across a quantum spin chain. Physical Review A, 84(5), [052316]. https://doi.org/10.1103/PhysRevA.84.052316

Published in:

Physical Review A

Document Version:

Publisher's PDF, also known as Version of record

Queen's University Belfast - Research Portal:

Link to publication record in Queen's University Belfast Research Portal

Publisher rights

(2011 American Physical Society

\section{General rights}

Copyright for the publications made accessible via the Queen's University Belfast Research Portal is retained by the author(s) and / or other copyright owners and it is a condition of accessing these publications that users recognise and abide by the legal requirements associated with these rights.

Take down policy

The Research Portal is Queen's institutional repository that provides access to Queen's research output. Every effort has been made to ensure that content in the Research Portal does not infringe any person's rights, or applicable UK laws. If you discover content in the Research Portal that you believe breaches copyright or violates any law, please contact openaccess@qub.ac.uk. 


\title{
Propagation of nonclassical correlations across a quantum spin chain
}

\author{
S. Campbell, ${ }^{1,2,3}$ T. J. G. Apollaro, ${ }^{4}$ C. Di Franco, ${ }^{2}$ L. Banchi, ${ }^{4,5}$ A. Cuccoli, ${ }^{4,5}$ R. Vaia, ${ }^{6}$ F. Plastina, ${ }^{7,8}$ and M. Paternostro ${ }^{1}$ \\ ${ }^{1}$ Centre for Theoretical Atomic, Molecular and Optical Physics, School of Mathematics and Physics, Queen's University, \\ Belfast UK BT7 INN, Ireland, United Kingdom \\ ${ }^{2}$ Physics Department, University College Cork, Cork, Republic of Ireland \\ ${ }^{3}$ Quantum Systems Unit, Okinawa Institute of Science and Technology, Okinawa, Japan \\ ${ }^{4}$ Dipartimento di Fisica e Astronomia, Università di Firenze, Via G. Sansone 1, IT-50019 Sesto Fiorentino (FI), Italy \\ ${ }^{5}$ INFN Sezione di Firenze, via G.Sansone 1, IT-50019 Sesto Fiorentino (FI), Italy \\ ${ }^{6}$ Istituto dei Sistemi Complessi, Consiglio Nazionale delle Ricerche, via Madonna del Piano 10, IT-50019 Sesto Fiorentino (FI), Italy \\ ${ }^{7}$ Dipartimento di Fisica, Università della Calabria, IT-87036 Arcavacata di Rende (CS), Italy \\ ${ }^{8}$ INFN Gruppo collegato di Cosenza, Universita della Calabria, IT-87036, Arcavacata di Rende (CS), Italy
}

(Received 3 June 2011; published 15 November 2011)

\begin{abstract}
We study the transport of quantum correlations across a chain of interacting spin- $1 / 2$ particles. As a quantitative figure of merit, we choose a symmetric version of quantum discord and compare it with the transported entanglement, addressing various operating regimes of the spin medium. Discord turns out to be better transported for a wide range of working points and initial conditions of the system. We relate this behavior to the efficiency of propagation of a single excitation across the spin chain. Moreover, we point out the role played by a magnetic field in the dynamics of discord in the effective channel embodied by the chain. Our analysis can be interestingly extended to transport processes in more complex networks and the study of nonclassical correlations under general quantum channels.
\end{abstract}

DOI: 10.1103/PhysRevA.84.052316

PACS number(s): 03.67.Hk, 03.65.Yz, 75.10.Pq, 42.50.Lc

The behavior of features such as quantum coherence and entanglement in a composite quantum system whose state is exposed to the effects of environmental actions has been the focus of an extensive research activity. Recently, much attention has been paid to the case of environments embodied by systems of interacting quantum particles [1]. Such dynamical environments can induce interesting back actions on the evolution of a system, thus significantly affecting its properties. From the point of view of coherent information processing, on the other hand, the nontrivial dispersion properties of networks of such interacting particles represent an interesting opportunity for their use as short-haul communication channels for the interconnections among onchip nodes in the next generation of information processing devices [2].

While most of the work in these contexts has focused on the study of the properties of entanglement upon propagation in such media, it is now widely accepted that the space of nonclassical correlations accommodates more than just quantum entanglement. Figures of merit such as quantum discord [3,4] and measurement-induced disturbance [5], to cite only two of the most popular ones, are able to capture the content of nonclassical correlations of a state well beyond entanglement. Although the role played by such broader forms of nonclassical correlations in the quantum-mechanical manipulation of information has yet to be fully understood, enormous is the interest they bring about as the manifestation of the various facets of quantumness in a system. It is thus very important to work on the exploration of the behavior of such quantities upon exposure to dynamical and finite environments of the sort addressed above, so as to build a useful parallel with the much more extensively investigated case of entanglement.

In this paper we study the propagation of quantum correlations across a system of interacting spin-1/2 particles. Our main goal is to compare the way important indicators of nonclassicality, such as quantum discord (QD) [3,4] and entanglement of formation (EOF) [6], are transferred through a medium offering nontrivial dispersion properties. In doing this, we aim at understanding whether or not the fundamentally conceptual difference between entanglement and discord leaves signatures in the way such nonclassical quantities are transferred. We show that this is indeed the case by preparing a nonseparable (in general mixed) state of an isolated spin and the one occupying the first site of a linear spin chain. We then compare the quantum-correlation properties of such an initial state with those of the state achieved, at a given instant of time of the evolution, between the isolated spin and the one occupying the last site of the chain itself. QD appears to be better transmitted than entanglement (as quantified by EOF) in a wide range of working conditions and regardless of the details of the initial state being considered. It is more robust to the dispersion inherent in the effective spin medium across which it propagates, being nonzero in situations where the EOF is, for all practical purposes, null. By relating the entanglement to the single-excitation transition amplitude of the system, we identify the working point at which a crossover occurs between the quality of transport of QD and EOF, making the transport of entanglement more efficient. Moreover, interesting effects of entanglement forerunning [7], where QD precedes the establishment of EOF, are found in the way quantum correlations build up between the isolated spin and the last one in a given chain. Our analysis considers a large number of state families in such a transport problem, addressing explicitly those that maximize the degree of discord at given global mixedness [8-10].

Our study provides exact quantitative answers to a problem that has been so far largely overlooked, although being relevant for a wide range of physical situations. For instance, it is sufficient to think about recent studies of the propagation of information in biological systems operating on the verge of 
quantumness, which appear to benefit from the inclusion of a mild degree of noise [11]. In such conditions, one might wonder whether other forms of quantum correlations are favored, given that the perfect transport of entanglement would be prevented.

The remainder of this paper is organized as follows. In Sec. I we describe the physical situation at hand and provide the general analytical form of the time-dependent density matrix describing the state of the isolated spin and the last one in a chain. Section II is devoted to a brief introduction to the quantitative indicators of quantum correlations adopted in this work. In Sec. III A we face the propagation of EOF and QD across the spin chain and address the relation between such nonclassicality indicators and the single-excitation transition amplitude. While the transport of discord appears to be favored, we point out the existence of a crossover point in the parameter space at which the performance of EOF becomes superior. Our study includes both pure and mixed input states, among them the case embodied by states that maximize QD at fixed global entropy. Section III B addresses the effect of a uniform magnetic field on the dynamics of the discord, finding that it has relevance only for states lacking rotational symmetry in the $x y$-spin plane, and, in Sec. III C, we describe briefly the evolution of the nonclassicality indicators under the influence of an environment modeled by a spin chain. Finally, in Sec. IV our conclusions are drawn and some open questions arising from the present work are put forward.

\section{THE MODEL}

We consider the configuration shown in Fig. 1, i.e., a quantum channel consisting of $N$ interacting spin- $1 / 2$ particles in a linear configuration with open boundary conditions. The Hamiltonian model describing the system is taken to be (we take units such that $\hbar=1$ throughout the manuscript)

$$
\hat{\mathcal{H}}=-2 J \sum_{i=1}^{N-1}\left(\hat{S}_{x}^{i} \hat{S}_{x}^{i+1}+\hat{S}_{y}^{i} \hat{S}_{y}^{i+1}\right)-2 h \sum_{i=1}^{N} \hat{S}_{z}^{i},
$$

with $\hat{S}_{k}^{i}$ being the $k=x, y, z$ spin-component operator of particle $i=1, \ldots, N, J$ being the interspin coupling strength, and $h$ being a uniform magnetic field. In what follows, the spins occupying sites $j=2, \ldots, N$ will be assumed to be all prepared in down state $|\downarrow\rangle$, with $\{|\downarrow\rangle,|\uparrow\rangle\}$ denoting the eigenstates of $\hat{S}_{z}$. On the other hand, the first spin forms a (generally mixed) bipartite quantum correlated state with a further particle, labeled 0 , which is physically detached from the chain.

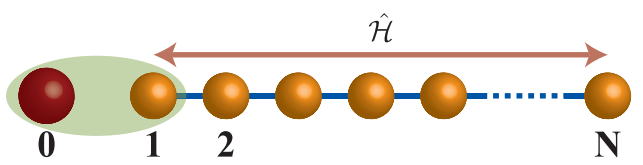

FIG. 1. (Color online) We consider a chain of $N$ interacting spin$1 / 2$ particles coupled through the Hamiltonian model $\hat{\mathcal{H}}$ [cf. Eq. (1)]. A further spin- $1 / 2$ particle, labeled 0 and completely isolated from the chain, is prepared in a joint mixed state $\rho^{(1,0)}(0)$ with particle 1 . We study how the general quantum correlations of such a state propagate across the chain.
The symmetry properties of the Hamiltonian model in Eq. (1) restrict the dynamics to those states within the zero- and the single-excitation sectors of the total Hilbert space of the spins [12], where the chain behaves as an amplitude damping channel [13] fully characterized by the transition amplitude of the spin excitation from site 1 to site $r=1, \ldots, N$. The latter is conveniently expressed as $f_{r}(t)=\left\langle r\left|e^{-i \hat{\mathcal{H}} t}\right| 1\right\rangle$, where in the states $|n\rangle(n=1, \ldots, N)$ all of the spins are in $|\downarrow\rangle$ except the one at position $n$, which is in $|\uparrow\rangle$.

In this basis, $\hat{\mathcal{H}}$ is represented by an $N \times N$ tridiagonal matrix, which can be analytically diagonalized for any length. The reduced density matrix $\rho^{(r)}(t)$ describing the state of particle $r$ at time $t$ can thus be expressed as a function of the state of particle 1 at the reference time $t=0$. That is,

$\rho^{(r)}(t)=\left[\begin{array}{cc}\rho_{\downarrow \downarrow}^{(1)}(0)+\rho_{\uparrow \uparrow}^{(1)}(0)\left(1-\left|f_{r}(t)\right|^{2}\right) & \rho_{\downarrow \uparrow}^{(1)}(0) f_{r}(t) \\ \rho_{\uparrow \downarrow}^{(1)}(0) f_{r}^{*}(t) & \rho_{\uparrow \uparrow}^{(1)}(0)\left|f_{r}(t)\right|^{2}\end{array}\right]$,

where $\rho_{\alpha \beta}=\langle\alpha|\rho| \beta\rangle(\alpha, \beta=\downarrow, \uparrow)$.

In the remainder of this work we will consider the chain as prepared in the fully factorized state $\otimes_{j=2}^{N}|\downarrow\rangle_{j}$, while the initial state of spins 0 and 1 is a quantum correlated state. Clearly, while spin 0 undergoes only a free evolution, the chain's elements evolve according to the intrachain coupling model in Eq. (1). This implies that the overall time evolution operator $\hat{\mathcal{U}}(t)$ that propagates the state of the $N+1$ spins factorizes as $\hat{\mathcal{U}}(t)=\hat{\mathbb{1}} \otimes e^{-i \hat{\mathcal{H}} t}$. Together with the uncorrelated initial state of the remaining part of the chain (which thus shares no correlation with spins 0 and 1), this legitimately allows us to make use of the formalism developed in Ref. [14] to get the joint state $\rho^{(r, 0)}(t)$ of spins $r$ and 0 from the knowledge of $\rho^{(r)}(t)$. Notice also that a very similar approach, which has also been verified by an exact numerical study, has been previously used in similar contexts [15]. Although such an approach is broadly valid and can indeed be used for any initial state of spins 0 and 1 , here we restrict our analysis to $X$-type input states of the general form

$\rho^{(1,0)}(0)=\left[\begin{array}{cccc}\rho_{11} & 0 & 0 & \rho_{14} \\ 0 & \rho_{22} & \rho_{23} & 0 \\ 0 & \rho_{23}^{*} & \rho_{33} & 0 \\ \rho_{14}^{*} & 0 & 0 & \rho_{44}\end{array}\right] \quad$ with $\quad \sum_{j=1}^{4} \rho_{j j}=1$.

Here we have introduced the compact notation $\rho_{i j}=\left\langle i\left|\rho^{(1,0)}(0)\right| j\right\rangle \quad$ with $\quad|1\rangle=|\downarrow \downarrow\rangle,|2\rangle=|\downarrow \uparrow\rangle,|3\rangle=$ $|\uparrow \downarrow\rangle$, and $|4\rangle=|\uparrow \uparrow\rangle$. In fact, as mentioned above, the excitation-preserving nature of the Hamiltonian studied here (which commutes with the total number of excitations in the system) ensures that the $X$-type character of any input state of the pair $(1,0)$ is preserved upon evolution. More explicitly, the only nonzero elements of the evolved density matrix will be

$$
\begin{aligned}
& \rho_{11}^{(r, 0)}(t)=\rho_{11}+\left[1-\left|f_{r}(t)\right|^{2}\right] \rho_{33}, \quad \rho_{33}^{(r, 0)}(t)=\left|f_{r}(t)\right|^{2} \rho_{33}, \\
& \rho_{22}^{(r, 0)}(t)=\rho_{22}+\left[1-\left|f_{r}(t)\right|^{2}\right] \rho_{44}, \quad \rho_{44}^{(r, 0)}(t)=\left|f_{r}(t)\right|^{2} \rho_{44}, \\
& \rho_{14}^{(r, 0)}(t)=f_{r}(t) \rho_{14}, \quad \rho_{23}^{(r, 0)}(t)=f_{r}(t) \rho_{23} .
\end{aligned}
$$


The initial conditions being specified by the input state, the above equations describe both the propagation of quantum correlations from site 1 to site $r$, when $r>1$, and the decohering influence of an environment (embodied by the spin chain) on the spin occupying site 1 , when $r=1$. In the former case, the single-excitation transition amplitudes $f_{r}(t)$ contain information on the working conditions of the channel. The limiting case $f_{r}(t)=1$ gives $\rho^{(r, 0)}(t)=\rho^{(1,0)}(0)$, i.e., the perfect transfer of the input state from pair $(1,0)$ to $(r, 0)$. On the other hand, $f_{1}(t)$ defines the probability amplitude of finding the excitation on the first spin. Note that $X$-type density matrices are such that no single-spin coherence will develop in time, i.e., both the reduced single-spin density matrices remain diagonal.

\section{FIGURES OF MERIT FOR QUANTUM CORRELATIONS}

After having introduced the dynamical model that will be addressed in our study, we turn our attention to the figures of merit that will be used in order to perform our quantitative analysis. As already anticipated, we take QD $[3,4]$ as a measure for general quantum correlations between any two spins under study. As originally proposed by Ollivier and Zurek [3], QD can be associated with the difference between two classically equivalent versions of mutual information, which measures the total correlations within a quantum state. For a two-spin state $\rho^{\left(r, r^{\prime}\right)}$ extracted from our system, the mutual information is defined as $\mathcal{I}\left[\rho^{\left(r, r^{\prime}\right)}\right]=\mathcal{S}\left[\rho^{(r)}\right]+\mathcal{S}\left[\rho^{\left(r^{\prime}\right)}\right]-\mathcal{S}\left[\rho^{\left(r, r^{\prime}\right)}\right]$. Here, $\mathcal{S}(\rho)=-\operatorname{Tr}\left(\rho \log _{2} \rho\right)$ is the von Neumann entropy of a generic state $\rho$. Alternatively, one can consider the one-way classical correlation $\mathcal{J}^{\leftarrow}\left[\rho^{\left(r, r^{\prime}\right)}\right]=\mathcal{S}\left[\rho^{(r)}\right]-\mathcal{H}_{\hat{\Pi}_{i}}\left(r \mid r^{\prime}\right)$ [4], where we have introduced $\mathcal{H}_{\hat{\Pi}_{i}}\left(r \mid r^{\prime}\right) \equiv \sum_{i} p_{i} \mathcal{S}\left(\rho_{r \mid r^{\prime}}^{i}\right)$ as the quantum conditional entropy associated with the the postmeasurement density matrix $\rho_{r \mid r^{\prime}}^{i}=\operatorname{Tr}_{r^{\prime}}\left[\hat{\Pi}_{i} \rho^{\left(r, r^{\prime}\right)}\right] / p_{i}$ obtained upon performing the complete projective measurement $\Pi_{i}$ on spin $r^{\prime}$, $p_{i}=\operatorname{Tr}\left[\hat{\Pi}_{i} \rho^{\left(r, r^{\prime}\right)}\right]$. QD is thus defined as

$$
\mathcal{D}^{\leftarrow}=\inf _{\left\{\Pi_{i}\right\}}\left[\mathcal{I}\left(\rho^{\left(r, r^{\prime}\right)}\right)-\mathcal{J}^{\leftarrow}\left(\varrho^{\left(r, r^{\prime}\right)}\right)\right]
$$

with the infimum calculated over the set of projectors $\hat{\Pi}_{i}$ [3]. Analogously, one can define $\mathcal{D}^{\rightarrow}$, which is obtained upon swapping the roles of $r$ and $r^{\prime}$. The inherently asymmetric definition of QD makes, quite naturally, $\mathcal{D}^{\rightarrow} \neq \mathcal{D}^{\leftarrow}$. This might be the cause of misinterpretations: a quantum-classical state for which $\mathcal{D}^{\leftarrow} \neq 0$ but $\mathcal{D}^{\rightarrow}=0$ (or vice versa) [16] might be interpreted as strictly classical if only $\mathcal{D}^{\rightarrow}\left(\mathcal{D}^{\leftarrow}\right)$ is probed. Here, we are interested in the transport of quantum correlations, regardless of the way in which they are encoded in the two-spin state. Rather refined solutions to this issue, passing through the generalization of the definition of QD or the introduction of strictly faithful entropic measures that are null only for classical-classical states (i.e., states such that $\mathcal{D}^{\leftarrow, \rightarrow}=0$ ), have been proposed [9]. However, they typically require a double optimization to be performed over a bilateral set of projective measurements. In order to bypass the numerical burden that this would imply, we consider the two-way QD:

$$
\mathcal{D}=\max \left[\mathcal{D}^{\leftarrow}, \mathcal{D}^{\rightarrow}\right]
$$

which is strictly null only on states endowed with no quantum correlations and faithfully signals classical-classical states [16].

On the other hand, our chosen entanglement measure is EOF [6], which quantifies the minimum number of Bell pairs needed in order to prepare a copy of the state $\rho^{\left(r, r^{\prime}\right)}$ we are studying. The relationship between EOF and QD has been recently examined to study the distribution of quantum correlated states in the entropic space [8]. Moreover, it is possible to establish a triangular relation connecting QD, EOF, and conditional entropy in multispin quantum states [17], so that such two figures of merit appear to be natural choices for a quantitative comparison. For arbitrary two-spin states, EOF is calculated as

$$
\mathcal{E}=h\left[\frac{1}{2}\left(1+\sqrt{1-C^{2}}\right)\right],
$$

where $h(x)=-x \log _{2} x-(1-x) \log _{2}(1-x)$ is the binary entropy function and $C$ is the concurrence of the state [6]. The latter, an equally valid entanglement measure, is found in terms of the eigenvalues $\lambda_{1} \geqslant \lambda_{2,3,4}$ of the matrix $\rho^{\left(r, r^{\prime}\right)}\left(\hat{\sigma}_{y} \otimes \hat{\sigma}_{y}\right) \rho^{\left(r, r^{\prime}\right) *}\left(\hat{\sigma}_{y} \otimes \hat{\sigma}_{y}\right)$ as

$$
C=\max \left[0, \sqrt{\lambda_{1}}-\sum_{i=2}^{4} \sqrt{\lambda_{i}}\right],
$$

where $\hat{\sigma}_{y}$ is the $y$-Pauli operator. For an $X$-type state of pair $\left(r, r^{\prime}\right)$, the concurrence is straightforwardly shown to be

$$
C_{\left(r, r^{\prime}\right)}=2 \max \left[0,\left|\rho_{14}\right|-\sqrt{\rho_{22} \rho_{33}},\left|\rho_{23}\right|-\sqrt{\rho_{11} \rho_{44}}\right] .
$$

Differently from EOF, QD does not have a closed analytical expression for any two-spin state, although some steps toward this goal have been performed [18,19]. Nevertheless, for the special case of the class of states presented in Eq. (3), one can obtain analytic formulas for $\mathcal{D}$ as a function of the dynamical parameter $f(t)$, as well as of the input state. However, as their expressions are lengthly and not very informative, we do not report them explicitly here.

\section{PROPAGATION OF QUANTUM CORRELATIONS}

\section{A. Case study of pure states and maximally discorded mixed states}

We perform our analysis by addressing the propagation of quantum correlations across the spin chain when the pair of spins $(1,0)$ is initialized in a given nonseparable state, while the remaining spins are in $\otimes_{j=2}^{N}|\downarrow\rangle_{j}$. In order to address the temporal behavior of our figures of merit, we need the explicit form taken by the single-excitation transition amplitude $f_{r}(t)$. Although the present formalism is valid without any major difficulties for general $r$ belonging to the chain, for the sake of clarity we set hereafter $r=N$ and omit the subscript in $f(t)$. For a uniform chain ruled by Eq. (1), such quantity reads

$$
f(t)=\frac{2}{N+1} \sum_{k=1}^{N} \sin \frac{k \pi}{N+1} \sin \frac{k \pi N}{N+1} \mathrm{e}^{-2 i t\left(h+\cos \frac{k \pi}{N+1}\right)} .
$$

We will use this explicit result to build up the state of spins 0 and $N$ and thus calculate quantum correlations. The 
single-excitation transition amplitude is a real (purely imaginary) quantity for $N$ odd (even), due to the symmetry properties of the spectrum of the system (a more detailed discussion on the properties of function $f(t)$ is given in Ref. [28]).

As a first significant instance, we consider the case in which the joint state of spins 0 and 1 is pure. In fact, for pure states, $\mathcal{E}=\mathcal{D}$, and it is interesting to study whether or not, in this case, entanglement is lost in favor of discord as information propagates across our dispersive medium. As the EOF is based on concurrence, we consider the class of pure entangled input states parameterized as

$$
\left|\psi\left(C_{(1,0)}\right)\right\rangle_{(1,0)}=\sin \gamma|\downarrow \downarrow\rangle+\cos \gamma|\uparrow \uparrow\rangle,
$$

where $\gamma=(1 / 2) \arcsin C_{(1,0)}$. The evolved state is then achieved using the approach outlined in Sec. I.

Perfect transfer of entanglement across an interacting spin chain is known to occur under proper conditions [20]. In particular, it was proved that perfect entanglement transfer is achieved when perfect end-to-end state transfer is made possible. For a system of three spins, the Hamiltonian model in Eq. (1) allows for perfect state transfer [21], thus implying that this is also the case for entanglement and QD, since for pure states $\mathcal{E}=\mathcal{D}$. Nonetheless, the examination of Fig. 2 shows some interesting features. Clearly, the peaks shown in all panels of Fig. 2 are achieved at the instants of time at which the input state (11) is perfectly transmitted, where the pair $(3,0)$ is pure and $\mathcal{E}=\mathcal{D}=\mathcal{E}_{(1,0)}$. However, between two consecutive peaks, the state of spins 0 and $N$ is mixed and the two figures of merit can be quantitatively different. Evidently, within these time windows the transport of QD is favored with respect to $\mathrm{EOF}$, being not only quantitatively larger than $\mathcal{E}$ but also non-null at times such that the EOF is, for all practical purposes, zero. Interestingly, as we increase the initial degree of entanglement of $\left|\psi\left[C_{(1,0)}\right]\right\rangle_{(1,0)}$, this effect becomes less important until, at $C_{(1,0)} \simeq 1$, there are narrow regions close to (a)

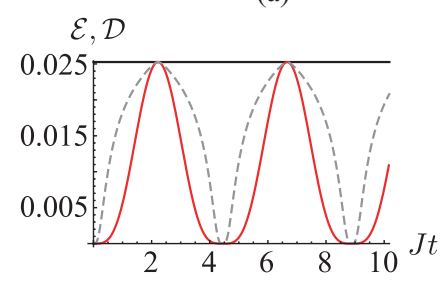

(c)

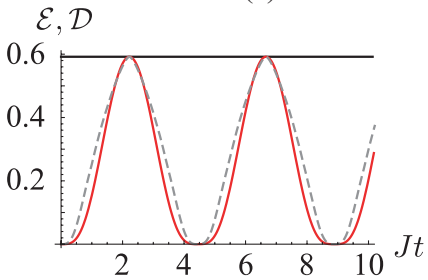

(b)

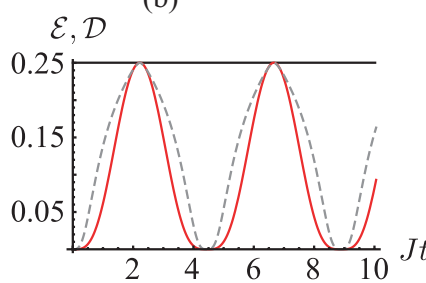

(d)

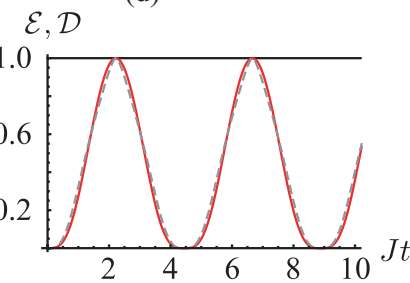

FIG. 2. (Color online) Behavior of quantum correlations between spins 0 and 3 against dimensionless time $J t$ in a system ruled by $\hat{\mathcal{H}}$ with uniform interaction strengths and arbitrary $h$. (a)-(d) $C_{(1,0)}=$ $0.1,0.4,0.7$, and 1 , respectively, with the initial EOF shown as a straight line. The solid (red) curve shows the EOF, while the dashed (gray) one is for the shared two-way QD. (a)

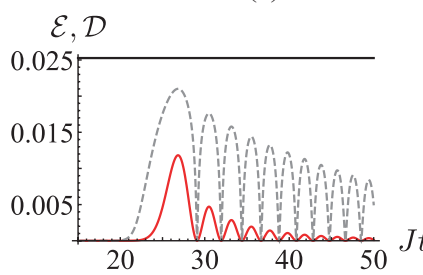

(c)

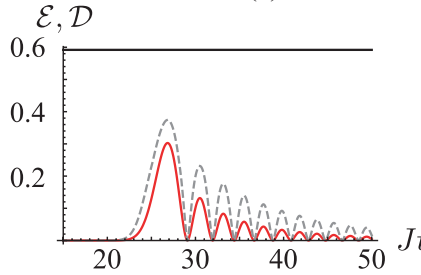

(b)

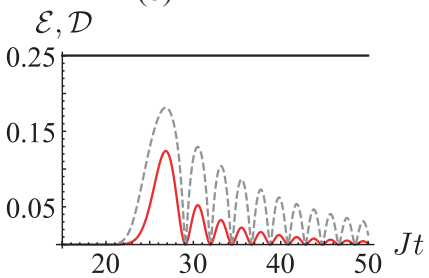

(d)
FIG. 3. (Color online) Behavior of quantum correlations between spin 0 and 50 against dimensionless time $J t$ for a system ruled by $\hat{\mathcal{H}}_{T}$ with arbitrary $h$. (a)-(d) $C_{(1,0)}=0.1,0.4,0.7$, and 1 , respectively, with the initial EOF shown as a straight line. The solid (red) curve shows the EOF $\mathcal{E}$, while the dashed (gray) one is for the shared two-way QD D.

the peaks where the transported entanglement overcomes QD. We will see later on in this paper how this effect depends on the single-excitation transition amplitude.

The behavior disclosed above becomes much more visible when we consider longer chains. The uniformity of the coupling strengths across the medium prevents perfect state transfer (and thus perfect entanglement transfer) for chains of more than three spins [20,21]. Therefore, we find an interesting feature in the corresponding propagation of $\mathcal{D}$ (see Fig. 3): the discrepancy between the propagated EOF and QD becomes quantitatively much more significant, while the degree of propagated discord is damped in time much more slowly than entanglement. However, such effects depend strongly on the initial value of entanglement in a way that the behaviors of $\mathcal{E}$ and $\mathcal{D}$ almost merge as $\mathcal{E}_{(1,0)} \rightarrow 1$. In Fig. 3 we show an instance of this case by reporting the propagation of $\mathcal{E}$ and $\mathcal{D}$ across a system of 50 spins interacting according to $\hat{\mathcal{H}}$.

Needless to say, as the two figures of merit refer to two different forms of quantum correlations, some quantitative differences should be expected. However, here we would like to stress that, the input state being pure, as remarked above, QD and EOF are exactly equivalent. This implies that the differences $|\mathcal{D}(t)-\mathcal{E}(0)|$ and $|\mathcal{E}(t)-\mathcal{E}(0)|$ faithfully quantify the performance of each nonclassicality indicator upon propagation.

These issues are better discussed by looking at Fig. 4, where we plot the propagated QD and EOF against time for a total of 15 spins. In each panel, the plane for which $\mathcal{E}(t)=\mathcal{D}(t), \forall t$ is displayed as a reference. Any point lying above the plane corresponds to discord overcoming EOF. Our choice of $N=15$ is only due to the clarity of the associated figures and does not hinder the validity of our conclusions.

We now show that the qualitative features revealed by our study on pure input states hold also when a mixed state of the spin pair $(1,0)$ is prepared. As in general the value of discord and entanglement associated to such input states will not coincide, this situation encompasses from the start the 
(a)

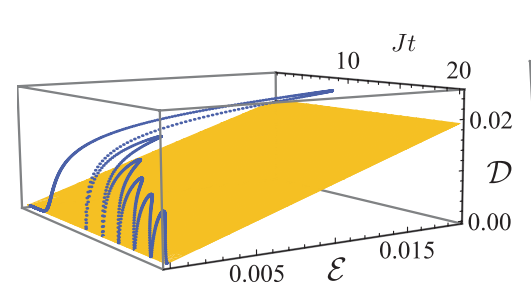

(b)

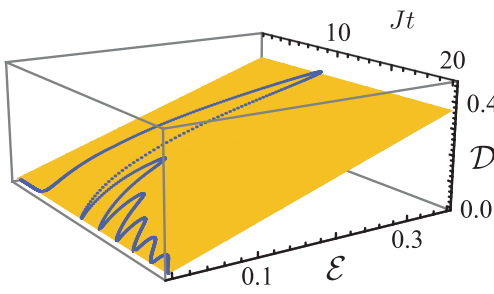

(c)

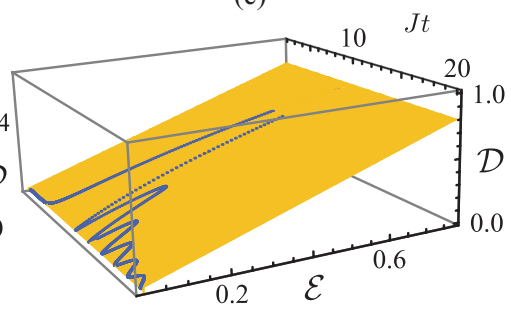

FIG. 4. (Color online) Behavior of QD against entanglement and dimensionless propagation time $J t$ for a chain of 15 spins, homogeneous intrachain couplings, and arbitrary $h$. The yellow plane at $\mathcal{D}=\mathcal{E}$ is used as a guide to the eye for discerning whether or not $\mathcal{D} \geqslant \mathcal{E}$. Each panel is for a different initial value of concurrence in the pair $(1,0)$. (a)-(c) $C_{(1,0)}=0.1,0.6$, and 1 , respectively. As the initial degree of entanglement grows, the efficiency of transport of $\mathcal{D}$ gets very close to that for $\mathcal{E}$.

profound differences between QD and EOF. In order to wash out the ambiguities associated with the possible choices for input mixed states, we refer to the studies in Refs. [8,9]. There, families of two-spin mixed states maximizing the degree of the two-way QD at fixed values of the global entropy have been identified and fully characterized. Furthermore, for these states $\mathcal{D}^{\leftarrow}=\mathcal{D}^{\rightarrow}$, so it is immaterial which spin is attached to the chain. We dub such states as maximally discorded mixed states (MDMS), which represent the counterpart, as far as discord is concerned, of the well-known maximally entangled mixed states (MEMS) [22]. Even more interesting, under proper choices of entanglement measures, part of the MEMS frontier is shared with the MDMS one [9]. As such extremal states are clearly dressed with a particular significance, we now restrict our study to them. The MDMS boundary is a patchwork of three classes of $X$-type states whose elements, following the notation used in Eq. (3), are given by

$$
\begin{array}{lll}
\rho_{11}^{P}=\rho_{14}^{P}=\frac{a}{2}, & \rho_{22}^{P}=\frac{1-a-g}{2}, & \rho_{33}^{P}=\frac{1-a+g}{2}, \\
\rho_{11}^{W}=\frac{1+a}{4}, & \rho_{22}^{W}=\rho_{33}^{W}=\frac{1-a}{4}, & \rho_{14}^{W}=\frac{a}{2}, \\
\rho_{11}^{R}=\frac{1-a}{2}, & \rho_{22}^{R}=a, \quad \rho_{33}^{R}=0, & \rho_{14}^{R}=\frac{g}{2},
\end{array}
$$

and $\rho_{23}^{P, W, R}=0$. While $\rho^{P}$ is a general two-parameter family $(a+g \leqslant 1), \rho^{W}(-1 / 3 \leqslant a \leqslant 1)$ are Werner states [23] and $\rho^{R}(0 \leqslant a \leqslant 1 / 3$ with $a+g \leqslant 1)$ are MEMS when the relative entropy is chosen as a measure of entanglement [22]. Such states belong to the MDMS frontier only under properly chosen values of $a$ and $g$. Such conditions are in general highly nontrivial, and we refer to Ref. [9] for full details. Here it is enough to state that we will consider values of such parameters that guarantee the MDMS nature of the corresponding states.

In order to provide a faithful evaluation of their performance upon propagation, we will compare the rescaled quantities $\tilde{\mathcal{R}}=\mathcal{R} / \mathcal{R}_{(1,0)}$ with $\mathcal{R}=\mathcal{E}, \mathcal{D}\left[\mathcal{R}_{(1,0)}=\mathcal{E}_{(1,0)}, \mathcal{D}_{(1,0)}\right]$ being the value of one of our figures of merit after propagation (for the initial spin pair). Moreover, rather than replicating the timedependent study performed so far and in order to provide a universal analysis freed from the choice of $N$, we will consider the propagated QD and EOF as general functions of the singleexcitation transition amplitude $|f| \in[0,1]$ (from now on we drop the label stating its dependence on time).

We start by studying Werner states, which are entangled only for $a \geqslant 1 / 3$. For values mildly larger than this threshold, where the purity of the state is small and also its entanglement is small, very large values of $|f|$ are required in order to actually transport $\mathcal{E}$. Differently, $\mathcal{D}$ is non-null for any $|f|$ and irrespectively of the initial QD properties of pair $(1,0)$. The relative discrepancy between the two figures of merit is in general very large and decreases only for almost ideal transport of excitations across the chain. As $a \rightarrow 1$, i.e., by increasing the purity of the state, more EOF is transported, even in the low- $f$ region, thus reducing the differences between the two nonclassicality indicators. In the limit of $a=1$, which makes $\rho^{W}$ a maximally entangled pure state, discord is overtaken by the EOF at $|f| \geqslant 1 / \sqrt{2}$. In fact, the state of pair $(N, 0)$ corresponding to such a value of the transition amplitude reads

$$
\tilde{\rho}^{W}=\left[\begin{array}{cccc}
\frac{1}{2} & 0 & 0 & \frac{1}{2 \sqrt{2}} \\
0 & \frac{1}{4} & 0 & 0 \\
0 & 0 & 0 & 0 \\
\frac{1}{2 \sqrt{2}} & 0 & 0 & \frac{1}{4}
\end{array}\right],
$$

which is an instance of a mixed state (its von Neumann entropy is 0.811278 ) with $\mathcal{D}=\mathcal{E}$. Figure 5 exemplifies the cases discussed above for three different values of parameter $a$.

When addressing the case of the two-parameter family $\rho^{P}$, the situation is even more striking. This class of states is MDMS for $g=0$ and $a \in[0.503,0.574]$, for instance, which correspond to a region of large mixedness such that the rescaled $\mathrm{QD}$ is always larger than the corresponding rescaled EOF, as (a)
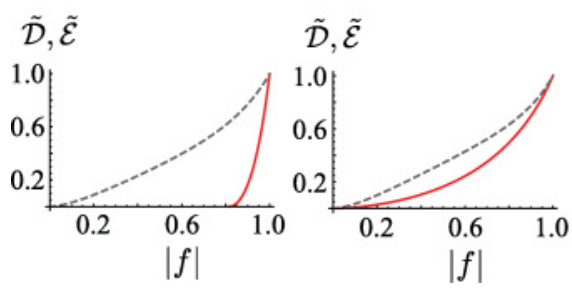

(c)

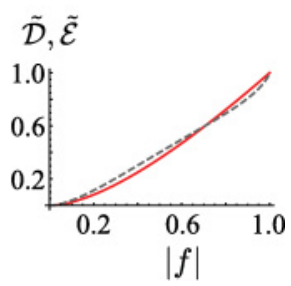

FIG. 5. (Color online) Comparison between the rescaled quantities $\tilde{\mathcal{D}}$ (gray dashed line) and $\tilde{\mathcal{E}}$ (red solid one) propagated across our chain of uniform couplings and the arbitrary magnetic field. The spin pair $(1,0)$ is prepared in a Werner state with $a=0.4,0.7$, and 1 [(a)-(c), respectively]. The curves are plotted against the transition amplitude $|f|$. 


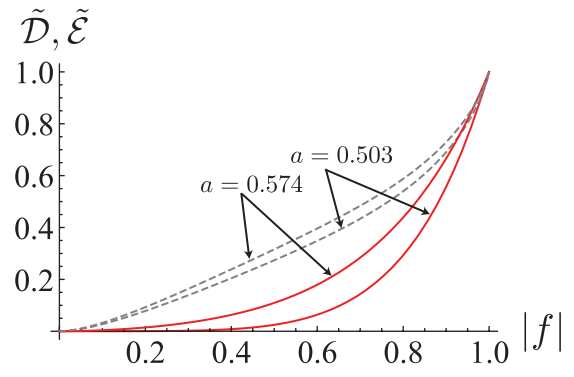

FIG. 6. (Color online) Comparison between the rescaled quantities $\tilde{\mathcal{D}}$ (gray dashed line) and $\tilde{\mathcal{E}}$ (red solid one) propagated across our chain of uniform couplings and the arbitrary magnetic field. The spin pair $(1,0)$ is prepared in state $\rho_{(1,0)}^{P}$ with $b=0$ and two values of $a$. The curves are plotted against the transition amplitude $|f|$.

seen in Fig. 6. In this case, $\tilde{\mathcal{E}}$ never overcomes $\tilde{\mathcal{D}}$ and can only equal it at $|f|=1$.

Finally, the general picture is confirmed by the investigation on the third family of boundary states, $\rho^{R}$, which are also the states maximizing the relative entropy of entanglement at fixed global mixedness [22]. The conditions that $a$ and $g$ should satisfy in order for $\rho^{R}$ to be a MDMS are rather nontrivial, passing through the solution of a transcendental equation [9]. For our purposes, it is enough to state that $\rho^{R}$ spans the largepurity region of the boundary and thus the crossing of QD and EOF at some value of $|f|$ should be expected. This is indeed the case, as highlighted in Fig. 7 for two values of parameters $a$ and $g$ that guarantee the MDMS nature of such class of states. While Fig. 7(a) refers to a large-purity case (the von Neumann entropy of the corresponding state is 0.159 , implying a small degree of mixedness) where EOF wins over QD at large enough $|f|$, Fig. 7(b) is for a much more mixed state. In this case, as expected from the analysis above, $\mathcal{D}$ is better transported at any dynamical condition.

\section{B. Effects of a magnetic field}

Here we study the effects of a uniform magnetic field $h$ on the transport of quantum correlations. From Eq. (10) we see that the single-excitation transition amplitude at $h \neq 0$ differs from that at $h=0$ only by an overall oscillating phase factor, $f^{(h)}(t)=e^{-i 2 h t} f^{(0)}(t)$. Consequently, by using the general form of an evolved state in Eq. (4) and the expression for the concurrence of $X$-type states given in Eq. (9), it is (a)

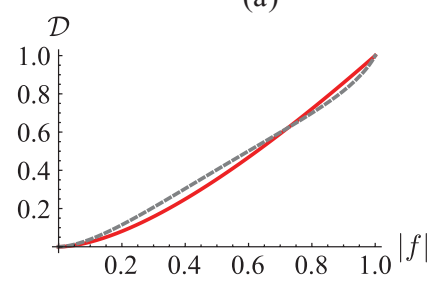

(b)

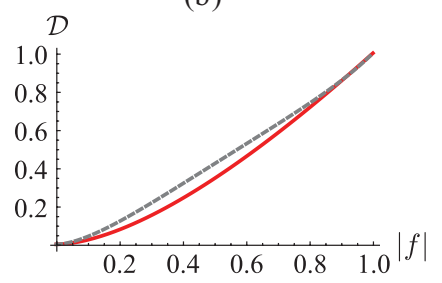

FIG. 7. (Color online) Comparison between the rescaled quantities $\tilde{\mathcal{D}}$ (gray dashed line) and $\tilde{\mathcal{E}}$ (red solid one) propagated across our chain of uniform couplings and no magnetic field. The spin pair $(1,0)$ is prepared in state $\rho_{(1,0)}^{R}$ with (a) $(a, g)=(0.0150,0.9747)$ and (b) $(a, g)=(0.1625,0.7649)$. The curves are plotted against the transition amplitude $|f|$. straightforward to see that $\mathcal{E}$ would depend on just $|f(t)|$ so that the introduction of a magnetic field does not affect the propagation of EOF.

The situation is radically different when considering the transport of QD for a certain class of input states of pair $(1,0)$. In fact, by considering states with maximally mixed marginals [5] $\rho^{(1,0)}(0)=\frac{1}{4}\left(\hat{\mathbb{1}}+\sum_{i=x, y, z} c_{i} \hat{\sigma}_{i} \otimes \hat{\sigma}_{i}\right)\left(c_{i} \in \mathbb{R}\right)$, for which analytic expressions for QD hold, we find that the magnetic field increases the amount of discord that can be obtained between 0 and $N$ as compared to the case with $h=0$. The reason for this enhancement can be found in the fact that, when $\left|c_{x}\right| \neq\left|c_{y}\right|, \mathcal{D}$ depends on both the real and imaginary part of $f$. Moreover, the phase factor $e^{-i 2 h t}$ in $f^{(h)}(t)$ yields an oscillating behavior of $\mathcal{D}$ that is lower bounded by the value achieved at $h=0$. In Fig. 8 we show the typical behavior described above.

$X$-type states with maximally mixed marginals allow for the identification of cases where the conditions for a truthful transport are breached. In all the cases studied so far, QD is a monotonically increasing function of $|f|$ with a maximum occurring at $t^{*} \sim N$, which are all features consistent with the picture of a transmission mechanism. For a maximally mixed marginal state having $\left|c_{x}\right| \neq\left|c_{y}\right|$, on the other hand, the discord between spin 0 and $N$ can be larger than the initial QD content of $\rho^{(1,0)}$. This point is best illustrated with the aid of an example. The density-matrix elements of a two-spin X-type state with zero discord should satisfy one of the following conditions $[9,24]$ :

(1) $\rho_{14}=\rho_{23}=0$. In this case, all the coherences are identically null and $\rho$ is purely diagonal. The corresponding state is thus a classical-classical one as $\mathcal{D}^{\leftarrow, \rightarrow}=0$.

(2) $\rho_{11}=\rho_{22}, \rho_{33}=\rho_{44}$ and $\left|\rho_{14}\right|=\left|\rho_{23}\right|$. This case corresponds to a quantum-classical state with $\mathcal{D}^{\rightarrow} \neq 0$ and $\mathcal{D}^{\leftarrow}=0$.

(3) $\rho_{11}=\rho_{33}, \rho_{22}=\rho_{44}$, and $\left|\rho_{14}\right|=\left|\rho_{23}\right|$, which give rise to a classical-quantum state with $\mathcal{D}^{\rightarrow}=0$ and $\mathcal{D}^{\leftarrow} \neq 0$.

When the symmetrized discord in Eq. (6) is used as a figure of merit, conditions 2 and 3 collapse into

(4) $\rho_{i i}=1 / 4(i=1, \ldots, 4)$ with $\left|\rho_{14}\right|=\left|\rho_{23}\right|$.
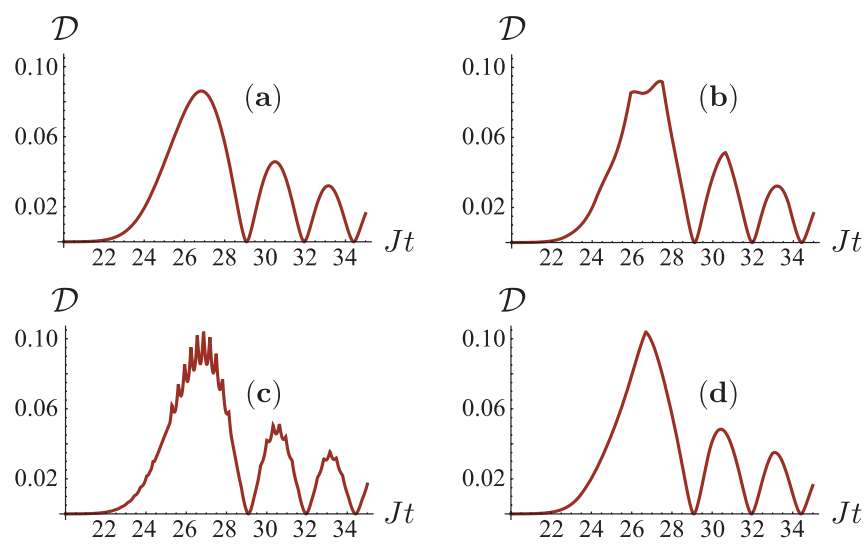

FIG. 8. (Color online) QD transported across a chain of 50 spins whose $(1,0)$ pair is initialized in an $X$-type state with maximally mixed marginals having $c_{x} \simeq 0.53, c_{y} \simeq 0.340$, and $c_{z} \simeq 0.035$. The associated value of $\mathrm{QD}$ is $\mathcal{D}_{(01)}(0)=0.210$. We have taken (a) $h / J=0$, (b) $h / J=0.5$, (c) $h / J=1$, and (d) $h / J=5$. 


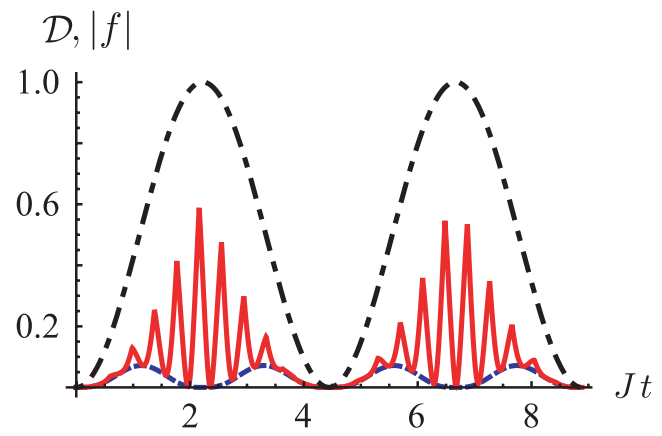

FIG. 9. (Color online) QD between spin 0 and 3, for a chain of $\mathrm{N}=3$ spins whose pair $(1,0)$ is initialized in an $X$-type state with maximally mixed marginals having $c_{x}=1, c_{y}=c_{z}=0$, and thus $\mathcal{D}(0)=0$. We have taken $h / J=0$ (blue dashed line) and $h / J=2$ (full red line). The dot-dashed line is the transition amplitude $\left|f_{3}(t)\right|$.

We now consider a $\rho^{(1,0)}(0)$ having $c_{y}=c_{z}=0$ and $c_{x}=1$, which corresponds to a state endowed with only classical correlations [embodied by $\left\langle\hat{\sigma}_{x}^{(1)} \otimes \hat{\sigma}_{x}^{(0)}\right\rangle \neq 0$ ]. The evolution yields a quantum-classical state:

$$
\rho^{(N, 0)}=\left(\begin{array}{cccc}
\frac{2-|f|^{2}}{4} & 0 & 0 & \frac{f}{4} \\
0 & \frac{2-|f|^{2}}{4} & \frac{f}{4} & 0 \\
0 & \frac{f}{4} & \frac{|f|^{2}}{4} & 0 \\
\frac{f}{4} & 0 & 0 & \frac{|f|^{2}}{4}
\end{array}\right) .
$$

If $f$ is a real (imaginary) function, such a state develops a nonzero correlation function $\left\langle\hat{\sigma}_{x}^{(N)} \otimes \hat{\sigma}_{x}^{(0)}\right\rangle\left[\left\langle\hat{\sigma}_{y}^{(N)} \otimes \hat{\sigma}_{x}^{(0)}\right\rangle\right]$ that is quantitatively equal to $f$ itself. However, spin $N$ also develops a nonzero magnetization in the $z$ direction given by $\left\langle\hat{\sigma}_{z}^{(N)}\right\rangle=1-|f|^{2}$. According to the results in Refs. [25,26], due to the noncommutativity between $\hat{\sigma}_{z}^{(N)} \otimes \hat{\mathbb{1}}^{(0)}$ and $\hat{\sigma}_{x}^{(N)} \otimes \hat{\sigma}_{x}^{(0)}$, the initially classical-type correlations acquire a quantum nature responsible for nonzero discord, as is shown in Fig. 9. Reasoning along the same lines, we can see that an initially zero-QD state fulfilling condition 1 remains such because all one- and two-spin correlators involve only $\hat{\sigma}_{z}$.

\section{Looking at the spin chain as a channel}

We now slightly change perspective and consider the spins occupying sites $j=2, \ldots, N$ as the elements of an environment for spin 1. This allows us to investigate the robustness of the quantum correlations shared with spin 0 under the influence of an amplitude damping channel. A similar analysis can be found in Ref. [27], where the entanglement dynamics in the presence of the same model has been studied. Here we extend this study to the analysis of QD so as to show that, at variance with entanglement, a unilateral nonunitary channel can induce quantum correlations in an initial state that is fully classical.

By using Eqs. (4) and (9), we obtain that entanglement sudden death (ESD) occurs when $\left|f_{1}(t)\right|^{2} \leqslant 1-\frac{\left|\rho_{14}\right|^{2}-\rho_{22} \rho_{33}}{\rho_{33} \rho_{44}}$ and $\left|f_{1}(t)\right|^{2} \leqslant 1-\frac{\left|\rho_{23}\right|^{2}-\rho_{11} \rho_{44}}{\rho_{33} \rho_{44}}$, for $\rho_{33} \rho_{44} \neq 0$. For states with initial finite entanglement, the quantities $\left|\rho_{14}\right|^{2}-\rho_{22} \rho_{33}$ and $\left|\rho_{23}\right|^{2}-$ $\rho_{11} \rho_{44}$ are always composed between 0 and 1 . For $\rho_{33}\left(\rho_{44}\right)=0$, the concurrence evolves as $C_{(1,0)}(t)=\left|f_{1}(t)\right| C_{(1,0)}(0)$. Besides this latter case, where ESD occurs only at $\left|f_{1}(t)\right| \equiv 0$, mixed nonseparable quantum states experience ESD due to the fact that appropriate choices of the interaction parameters make $\left|f_{1}(t)\right|$ range from 0 to 1 : The amplitude damping channel, acting only on one spin, is able to erase completely the quantum correlations which give rise to entanglement.

Now let us turn our attention to the conditions for the vanishing of QD discussed previously. As far as condition 1 is concerned, we observe that a zero-QD state will remain such at all times because an environment addressing only one spin of a bipartite state cannot build up quantum coherences. If the latter are initially present in such a way to start from a non-zero-QD state, condition 1 can be possibly fulfilled only asymptotically. On the other hand, starting from a zero-QD state according to condition 4 , we note that the constraint $\rho_{i i}=1 / 4$ breaks down because of the dynamics embodied by Eqs. (4) and the classical-classical state evolves into a quantum-classical one, so that $\mathcal{D}^{\rightarrow} \neq 0$. Conversely, starting from a state with nonzero $\mathrm{QD}$, it is necessary, in order to satisfy condition 4 , that the initial state consists of a quantum-classical state (condition 2) and that the environment acts on the spin that, when subjected to a complete projective measurement, gives rise to nonzero one-way QD. Furthermore, as the amplitude damping channel implies $\rho_{11}(t) \geqslant \rho_{11}(0)$, only quantum-classical states with $\rho_{11}(0)<\frac{1}{4}$ can evolve to a classical-classical one. This will occur at times $t^{*}$ such that $f_{1}\left(t^{*}\right)=1 / 2+\rho_{11} /\left(1-2 \rho_{11}\right)$.

\section{CONCLUSIONS}

We have studied the propagation of quantum correlations across a chain of interacting spins by looking at the performance of two significant figures of merit: quantum discord and entanglement of formation. The amount of transported quantum correlations has been quantified when the chain is seeded with various instances of two-spin mixed states. We have explicitly considered the case of pure entangled states, as well as the members of the boundary family of MDMSs, which maximize QD at set values of global entropy. Discord appears to be consistently better transported than entanglement, except for highly pure input states whose EOF is transported across a chain guaranteeing a large singleexcitation transition amplitude. Moreover, we have performed a brief case study on the conditions under which QD is actually created upon propagation across the chain, pointing toward the class of states giving rise to this effect and analyzing, in particular, how the discord increases under the effect of a magnetic field. Finally, we have revealed that, whereas the EOF of all mixed states undergoes ESD by an appropriate choice of the interaction parameters, QD vanishes only under very specific initial-state conditions and interaction settings. In particular, quantum-quantum states allow for vanishing discord under the influence of the spin environment considered here only asymptotically in time.

Our work extends the investigations performed so far on the propagation of entanglement to the broader realm of more general quantum correlations proving that, in the dispersive medium consisting of interacting quantum particles, correlations beyond entanglement are favored. Such results motivate the study on a few aspects of this general problem that still remain to be addressed, such as the extension to other quantifiers of nonclassicality, their behavior under more 
general dynamical conditions of the spin media, and the quantification of the corresponding non-Markovian effects brought about by the spin chain on the dynamics of general indicators of quantumness.

\section{ACKNOWLEDGMENTS}

We thank Marco Piani and Paola Verrucchi for discussions. T.J.G.A. is grateful to the Centre for Theoretical Atomic, Molecular, and Optical Physics at the School of
Mathematics and Physics, Queen's University Belfast, for hospitality during completion of this work. We acknowledge financial support from the Department of Employment and Learning, the Italian Ministry of Education, University, and Research through the 2008 PRIN program (Contract No. 2008PARRTS003); the Irish Research Council for Science, Engineering, and Technology; and the UK EPSRC (No. EP/G004579/1). M.P. and F.P. acknowledge support by the British Council/MIUR British-Italian Partnership Program 2009-2010.
[1] D. Rossini T. Calarco, V. Giovannetti, S. Montangero, and R. Fazio, Phys. Rev. A 75, 032333 (2007); D. Rossini, P. Facchi, R. Fazio, G. Florio, D. A. Lidar, S. Pascazio, F. Plastina, and P. Zanardi, ibid. 77, 052112 (2008); L. Amico, R. Fazio, A. Osterloh, and V. Vedral, Rev. Mod. Phys. 80, 517 (2008).

[2] S. Bose, Contemp. Phys. 48, 13 (2007).

[3] H. Ollivier and W. H. Zurek, Phys. Rev. Lett. 88, 017901 (2001).

[4] L. Henderson and V. Vedral, J. Phys. A 34, 6899 (2001).

[5] S. Luo, Phys. Rev. A 77, 042303 (2008).

[6] C. H. Bennett, D. P. DiVincenzo, J. A. Smolin, and W. K. Wootters, Phys. Rev. A 54, 3824 (1996); W. K. Wootters, Phys. Rev. Lett. 80, 2245 (1998).

[7] A. Auyuanet and L. Davidovich, Phys. Rev. A 82, 032112 (2010).

[8] A. Al-Qasimi and D. F. V. James, Phs. Rev. A 83, 032101 (2011).

[9] D. Girolami, M. Paternostro, and G. Adesso, J. Phys. : Math. Theor. 44, 352002 (2011).

[10] F. Galve, G. L. Giorgi, and R. Zambrini, Phys. Rev. A 83, 012102 (2011).

[11] M. B. Plenio and S. F. Huelga, New J. Phys. 10, 113019 (2008); P. Rebentrost, M. Mohseni, I. Kassal, S. Lloyd, and A. AspuruGuzik, ibid. 11, 033003 (2009); F. Caruso, A. W. Chin, A. Datta, S. F. Huelga, and M. B. Plenio, J. Chem. Phys. 131, 105106 (2009); A. W. Chin, A. Datta, F. Caruso, S. F. Huelga, and M. B. Plenio, New J. Phys. 12, 065002 (2010).

[12] W. Son, L. Amico, F. Plastina, and V. Vedral, Phys. Rev. A 79, 022302 (2009).

[13] S. Bose, Phys. Rev. Lett. 91, 207901 (2003).

[14] B. Bellomo, R. LoFranco, and G. Compagno, Phys. Rev. Lett. 99, 160502 (2007).
[15] S. Bose, Phys. Rev. Lett. 91, 207901 (2003).

[16] M. Piani, P. Horodecki, and R. Horodecki, Phys. Rev. Lett. 100, 090502 (2008).

[17] F. F. Fanchini, M. F. Cornelio, M. C. de Oliveira, and A. O. Caldeira, Phys. Rev. A 84, 012313 (2011); L.-X. Cen, X.-Q. Li, J. Shao, and Y. Yan, Phys. Rev. A 83, 054101 (2011).

[18] M. Ali, A. R. P. Rau, and G. Alber, Phys. Rev. A 81, 042105 (2010).

[19] D. Girolami and G. Adesso, Phys. Rev. A 83, 052108 (2011).

[20] A. Kay, Int. J. Quant. Inf. 8, 641 (2010).

[21] M. Christandl, N. Datta, A. Ekert, and A. J. Landahl, Phys. Rev. Lett. 92, 187902 (2004); M. Christandl, N. Datta, T. C. Dorlas, A. Ekert, A. Kay, and A. J. Landahl, Phys. Rev. A 71, 032312 (2005); F. Plastina and T. J. G. Apollaro, Phys. Rev. Lett. 99, 177210 (2007).

[22] W. J. Munro, D. F. V. James, A. G. White, and P. G. Kwiat, Phys. Rev. A 64, 030302 (2001); T. C. Wei, K. Nemoto, P. M. Goldbart, P. G. Kwiat, W. J. Munro, and F. Verstraete, ibid. 67, 022110 (2003)

[23] G. Vidal and R. F. Werner, Phys. Rev. A 65, 032314 (2002).

[24] G.-X. Li, Z. Yi, and Z. Ficek, e-print arXiv:1101.4983v1.

[25] A. Ferraro, L. Aolita, D. Cavalcanti, F. M. Cucchietti, and A. Acín, Phys. Rev. A 81, 052318 (2010).

[26] B. Dakic, V. Vedral, and C. Brukner, Phys. Rev. Lett. 105, 190502 (2010).

[27] T. J. G. Apollaro, A. Cuccoli, C. DiFranco, M. Paternostro, F. Plastina, and P. Verrucchi, New J. Phys. 12, 083046 (2010).

[28] L. Banchi, T. J. G. Apollaro, A. Cuccoli, R. Vaia, and P. Verrucchi, Phys. Rev. A 82, 052321 (2010); A. Bayat, L. Banchi, S. Bose, and P. Verrucchi, Phys. Rev. A 83, 062328 (2011). 